\title{
DECISION SUPPORT SYSTEM FOR BREAST CANCER DIAGNOSIS BY A META-LEARNING APPROACH BASED ON GRAMMAR EVOLUTION
}

\author{
Albert Fornells-Herrera, Elisabet Golobardes-Ribé and Ester Bernadó-Mansilla \\ Research Group in Intelligent Systems \\ Enginyeria i Arquitectura La Salle, Ramon Llull University. Quatre Camins 2, 08022 Barcelona (Spain)
}

\author{
Joan Martí-Bonmatí \\ Computer Vision and Robotics Group \\ University of Girona. Avda. Lluís Santaló s/n, 17071 Girona (Spain)
}

\begin{abstract}
Keywords: Breast Cancer Diagnosis, Strategic Decision Support Systems, Evolutionary Programming, Meta-Learning, Application of Artificial Intelligence on Medicine.

Abstract: The incidence of breast cancer varies greatly among countries, but statistics show that every year 720,000 new cases will be diagnosed world-wide. However, a low percentage of women who suffer it can be detected using mammography methods. Therefore, it is necessary to develop new strategies to detect its formation in early stages. Many machine learning techniques have been applied in order to help doctors in the diagnosis decision process, but its definition and application are complex, getting results which are not often the desired.

In this article we present an automatic way to build decision support systems by means of the combination of several machine learning techniques using a Meta-learning approach based on Grammar Evolution (MGE). We will study its application over different mammographic datasets to assess the improvement of the results.
\end{abstract}

\section{INTRODUCTION}

Breast cancer is the most common cancer among western women and is the leading cause of cancerrelated death in women aged 15-54. Screening programs have proved to be good practical tools for prematurely detecting and removing breast cancer, and increasing the survival percentage in women (Winfields et al., 1994). In an attempt to improve early detection, a number of Computer Aided Diagnosis (CAD) techniques have been developed. There are several approaches to CAD, but we focus on the breast cancer diagnosis using mammographic images. A mammographic image is processed in order to identify the microcalcifications $(\mu \mathrm{Ca})$ that appear. $\mathrm{Hu}-$ man experts agree on their relevance in diagnosing a new case. After characterizing the $\mu \mathrm{Ca}$ through a set of features, we diagnose each image using machine learning techniques. Previous studies applying machine learning techniques found that these techniques improved the accuracy rate (in terms of correct classifications) but decreased the reliability rate (in terms of robustness and stability) compared to human experts (Golobardes et al., 2002). Our purpose is to improve the reliability rate so experts can have more confidence in the results, when they need to decide whether a sample is benign or malign.

When people make critical decisions, they usually take into account the opinions of several experts rather than relying on their own judgement or that of an only trusted advisor. Therefore, an obvious approach for making more reliable decisions is to combine the output of several models/classifiers by means of a metalevel, which coordinates the decision support system. Although a $100 \%$ of reliability is not assured, the confidence in the results is usually increased.

However, models' combination has the disadvantage of being rather hard to design as it is not easy to intuitively understand what factors are contributing to the different predictions. An automatic process that searched for the best combination of single classifiers would help in the design process. In this paper, we propose an automatic way of defining decision support systems using a Meta-learning approach based on Grammar Evolution (MGE). Grammar Evolution (GE) (Ryan et al., 1998) is a variant of Evolutionary Computation (EC) (Goldberg, 1989) designed to find algorithms using a genotype to fenotype mapping process by means of a Backus Naur Form (BNF) grammar, which leads the searching process. We adapt a GE to guide the search to the most reliable schema of classifier combinations. Also, the result- 
ing meta-learning approach will be applied to breast cancer diagnosis.

This article is organized as follows. Section 2 surveys some related work. Section 3 sets the background of classifier combination, and defines the main structure of our meta-learning approach (MGE). Section 4 describes the particular setting of MGE for the breast cancer diagnosis problem. Next, Section 5 analyzes the results and finally, we summarize conclusions and further work in section 6.

\section{RELATED WORK}

Several decision support systems have been applied to perform the diagnosis of breast cancer using the $\mu \mathrm{Ca}$ extracted from mammographic images. Some of them are Support Vector Machines (Campani et al., 2000), Nearest-Neighbour algorithms (Kauffman et al., 2000), Bayesian Networks (Edwards et al., 2000) or Fuzzy Neural Networks (Cheng et al., 1998).

We have been working successfully with different Artificial Intelligence (AI) techniques such as Genetic Algorithms (GA) (Goldberg, 1989) and Case Base Reasoning (CBR) (Aamodt and Plaza, 1994) to tackle this problem (Garrell et al., 1999) (Golobardes et al., 2002). However, we prefer to focus in a CBR approach because it allows experts to get an explanation of its classification (malign or benign) in terms of the most similar cases. In these previous works, the results were compared with the classifications by human experts (Martí et al., 2000). The conclusions were that AI techniques improved the accuracy rate but the reliability rate was decreased. One of the reasons was the difficulty of defining a reliable similarity function for CBR.

In (Golobardes et al., 2001) a Genetic Programming (GP) (Koza, 1992) approach was used as an automatic process for designing similarity functions for CBR. The system found a similarity function that improved the previous results, but they were still not good enough. The reason was attributed to the huge search space in which the GP had to find the solution. In (Fornells et al., 2005b) a new approach based on GE and CBR was proposed to reduce the search space, by the use of a grammar that led the search process. The comparison of the GP-CBR approach and the GE-CBR approach showed that the GE-CBR approach works better if the grammar is well defined (Fornells et al., 2005a).

Combining multiple models is a popular research topic in machine learning research. The most important methods for combining models are bagging (Breiman, 1996a), boosting (Schapire et al., 1997) and stacking (Wolpert, 1990). Bagging and boosting are based in the combination of their outputs using voting schemes. The difference between them is that boosting uses a weighting vote. On the other hand, Stacking was introduced by Wolpert (Wolpert, 1990) in the neural network literature, and it was applied to numeric prediction by Breiman (Breiman, 1996b). It is a technique based on a meta-level that makes decisions using heuristics which combine the outputs of several classifiers. Later, Ting and Witten (Ting and Witten, 1997a) compared different meta-level models empirically and found that a simple linear model performs best. Also, they demonstrated the advantage of using the probabilities of classifier predictions as meta-level data. A combination of stacking and bagging was also investigated in (Ting and Witten, 1997b). Many different models were generated by varying the learning parameters (Oliver and Dowe, 1995) (Kwok and Carter, 1990) and by using different sampling methods (Freund and Schapire, 1996) (Ali and Pazzani, 1996).

In (Vallespí et al., 2002) these concepts were used to define several meta-levels by means of heuristics based on the results of different machine learning techniques over the mammography dataset proposed in (Martí et al., 2000). Nevertheless, this way of defining meta-levels is very limited. For this reason, we propose MGE as an automatic way of defining metalevels, and we study its application over new and improved datasets of breast cancer diagnosis.

\section{MGE: META-LEARNING APPROACH BASED ON GRAMMAR EVOLUTION}

\subsection{Meta-learning}

Meta-learning can be defined as learning from information generated by a(some) learner(s). It can also be viewed as learning meta-knowledge from the learned information. Therefore, it is a general technique to coalesce the results of multiple classifiers.

It requires at least two levels: A level composed by a set of trained classifiers (level-0 model) using a subset of the original dataset (level-0 data), and another level trained (level-1 model) using the outputs of the level-0 models (level-1 data). (Breiman, 1996a) demonstrated that the combination of all the model- 0 outputs usually improves the results of the individual classifiers.

Level-0 models can be of two types: (1) Heterogeneous (the classifiers used are different) and (2) Homogeneous (all the classifiers used are equal). In turn, level-0 data can be distributed by each classifier in several ways: (1) Duplicating all the samples, (2) Distributing samples clustered in disjoint subsets, or (3) 
Distributing samples clustered in subsets allowing the repetition of the samples. Breiman (Breiman, 1996a) exposed that it is desired to level-0 models to be unstable, which means that they should be easily altered if their training dataset is altered. Nevertheless, Ting and Witten in (Ting and Witten, 1997b) demonstrated that it is not necessary. However, they both agree that the level-0 design process is critical in the sense that all classifiers must complement each other, that is, they need to cover all the possible solutions.

The first difficulty in the level-1 design process is to define what data from the output of level-0 classifiers should be used: (1) Only the class predicted (Breiman, 1996a), (2) The class predicted and the most similar samples (Chan and Stolfo, 1993), or (3) the probabilities of belonging to each possible class (Ting and Witten, 1997a). Option (1) does not allow level-1 model to get any measure of the confidence on the whole prediction. Although option (2) adds extra information using the internal information of level-0 models, it is difficult to integrate different internal representations. On the other hand, option (3) provides level-1 model information on the confidence in all the class predictions, which can be used to better evaluate the behaviour of the level-0 models. Nevertheless, the selection of the type of information used as level-1 data is conditioned by the level-1 model. There are several ways of implementing the level-1, of which the most important are: (1) Manual heuristics defined by an expert, (2) Voting schemas (Breiman, 1996a), (3) Weighting voting schemas (Schapire et al., 1997), (4) Arbiter strategy based on solving conflicts (Chan and Stolfo, 1993), (5) Lineal regressions schemes (Ting and Witten, 1997a) and (6) Inductive and explanation-based learning (Flann and Dietterich, 1989).

We can see that meta-learning allows the definition of a hierarchy, which can be used to model a decision support system.

\subsection{Grammar Evolution}

GE (Ryan et al., 1998) is a technique based on Evolutionary Computation (EC), where a BNF grammar is used in a genotype to fenotype mapping process in order to transform the individual (represented by an array of bits) into an executable program or function. The fitness is assigned depending on the result of the program.

The BNF grammar is composed by a tuple $\{\mathrm{N}, \mathrm{T}, \mathrm{P}$, $\mathrm{S}\}$. N and T represent the set of non-terminals and terminals respectively, $\mathrm{S}$ is the starting production, and $\mathrm{P}$ defines the rules for each production of the nonterminals. At the beginning of the mapping process, each individual has a program represented by the nonterminals of the starting production. The first step consists of clustering the bits of the individual in inte-

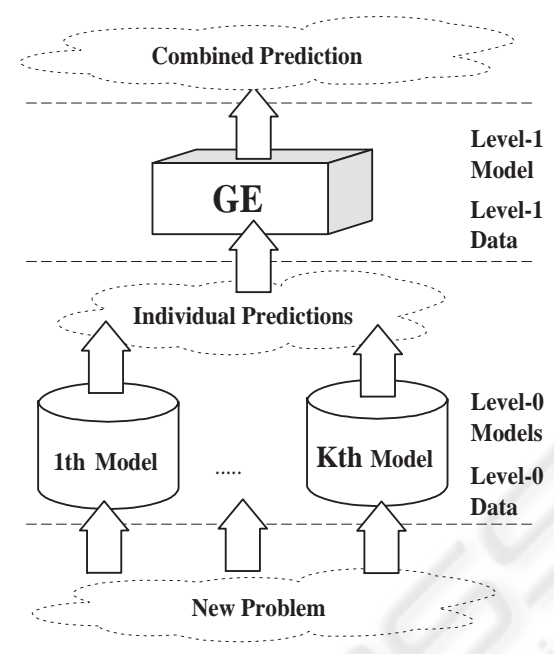

Figure 1: Evaluation of an individual in MGE.

gers of $\mathrm{X}$ bits called codons, where $\mathrm{X}$ depends on the production with more rules. Next, the non-terminals elements are iteratively replaced by the elements of one rule of the same production, which is selected using the codons of the individuals by the equation:

$$
\text { new rule }=\text { modulus of } \frac{\text { actual codon }}{\text { number of rules }}
$$

This process is repeated until all the elements of the program are terminals, and therefore the program can be run. If the codons have run out and the mapping process has not ended, then a wrapping operator is applied. It means that codons are reused again from the beginning.

\subsection{Definition of Meta-levels by Means of GE}

Subsection 3.1 describes the term meta-learning as a system which learns from other learning systems. Nevertheless, it is not trivial to define how to combine the output of the level- 0 models to get the level-1 model. For this reason, we want to automate it using the GE approach, which needs the definition of the BNF grammar and the evaluation of the individuals.

The BNF grammar can be defined as an expression that represents a linear regression, a set of rules, or any way of combining the outputs of several level-0 models. This variety allows GE more flexibility.

Figure 1 shows the evaluation of a sample by an individual (a potential meta-level), which consists of: (1) Translation of the individual into an expression modeled by the BNF grammar, (2) Test the sample in all level-0 models in order to get the level-1 data, (3) The last predictions are replaced in the expression 
Table 1: Characteristics of the datasets studied.

\begin{tabular}{|l|l|l|}
\hline Dataset & Attr. & Class Distribution \\
\hline Wisconsin & 10 & benign (458), malign (241) \\
\hline$\mu$ Ca & 22 & benign (121), malign (95) \\
\hline DDSM & 143 & $\begin{array}{l}\text { b1(61), b2(185), b3(157), } \\
\text { b4(98) }\end{array}$ \\
\hline MIAS-Birads & 153 & $\begin{array}{l}\text { b1(128), b2(78), b3(70), } \\
\text { b4(44) }\end{array}$ \\
\hline MIAS-3C & 153 & $\begin{array}{l}\text { fatty(106), dense(112), } \\
\text { glandular(104) }\end{array}$ \\
\hline
\end{tabular}

to get the combined prediction. Finally, the fitness of the individual is computed using the statistics over all the tested samples.

\section{SETTING MGE FOR THE PROBLEM}

\subsection{Datasets}

We applied our approach to different datasets related with breast cancer diagnosis (see Table 1). The Wisconsin dataset comes from UCI repository (Blake and Merz, 1998) and the rest of the them belong to our own repository. These are mammographic images digitalized by the Computer Vision and Robotics Group from the University of Girona. The $\mu \mathrm{Ca}$ dataset (Martí et al., 2000) contains samples from Trueta Hospital (in Girona), while DDSM (Heath et al., 2000) and MIAS (Suckling et al., 1994) are public mammographic images datasets, which have been studied and preprocessed in (Oliver et al., $2005 \mathrm{~b}$ ) (Oliver et al., 2005a) respectively. The $\mu \mathrm{Ca}$ dataset contains samples of mammographies previously diagnosed by surgical biopsy, which can be benign or malign. DDSM and MIAS-Bi classify mammography densities, which was found relevant for the automatic diagnosis of breast cancer. Experts classify them either in four classes (according to BIRADS (Samuels, 1998) classifications) or three classes (classification used in Trueta Hospital).

\subsection{Level-0 Model}

As we mentioned previously, we focus in CBR because it provides an explanation of its classification. In order to select the most suitable level-0 models, a previous study testing several techniques is needed over the different datasets. A self CBR approach with different configurations was tested with different similarity functions (Clark, Cosines, Hamming, Euclidean, Cubic) using sample correlation as weighting schema, and three nearest neighbour technique in the retrieval phase. Other machine learning techniques from Weka (Witten and Frank., 2000) were also tested: IBK (Aha and Kibler, 1991), ID3 (Quinlan, 1986), C4.5 (Quinlan, 1993), PART (Frank and Witten, 1998), Bayesian Neural Network (BNN) (Freeman and Skapura, 1991) and Sequential Minimal Optimization (SMO) (Platt, 1998).



Figure 2: BNF grammar used in GE.

Table 2 summarizes the error rates and the standard deviation for each dataset. The results were computed using a 10-fold stratified cross-validation, and using 10 random seeds. Because we want to improve the results for all datasets, the level- 0 models selected are these which have the lowest error and lowest standard deviation. They are marked using bold letters.

\subsection{Level-1 Model}

As we explained in subsection 3.1, the most useful level-1 data is the probability of belonging to each possible class. In the CBR approach, it is computed using the equation proposed by (Ting and Witten, 1997a):

$$
P_{i} x=\frac{\sum_{s=1}^{p} f\left(y_{s}\right) / d\left(x, x_{s}\right)}{\sum_{s=1}^{p} 1 / d\left(x, x_{s}\right)}
$$

where:

$$
\begin{aligned}
& x \text { is the new problem to solve } \\
& x_{s} \text { is the case 's' retrieved } \\
& d\left(x, x_{s}\right) \text { is the difference between ' } \mathrm{x} \text { ' and ' } x_{s} \text { ' } \\
& p \text { indicates the number of samples returned } \\
& f\left(y_{s}\right) \text { is ' } 1 \text { ' if } i=y_{s} \text {, and ' } 0 \text { ' otherwise }
\end{aligned}
$$

The probabilities for the other methods are computed internally by Weka. We define the level-1 data as a list of predictions generated by the samples tested, where each prediction is composed by the probabilities of belonging to each possible class (C), for each level-0 model used. 
Table 2: $\%$ of error and standard deviation using several machine learning over the datasets of the table 1 .

\begin{tabular}{|l|ccccc|}
\hline Method & Wisconsin & $\mu$ Ca & DDSM & MIAS-Bi & MIAS-3C \\
\hline Clark & $10.80(9.23)$ & $34.26(4.6)$ & $\mathbf{4 4 . 7 1 ( 6 . 6 )}$ & $\mathbf{2 6 . 8 8 ( 7 . 1 5 )}$ & $\mathbf{2 2 . 6 1}(\mathbf{8 . 4})$ \\
Cosines & $34.5(1.4)$ & $43.98(9.4)$ & $88.42(6.74)$ & $85.63(7.2)$ & $81.37(9.2)$ \\
Hamming & $3.43(1.3)$ & $\mathbf{3 2 . 4 1}(\mathbf{9 . 5 4})$ & $55.09(5.9)$ & $33.13(6.4)$ & $32.30(5.9)$ \\
Euclidean & $\mathbf{3 . 4 2 ( 1 . 2 )}$ & $34.72(7.14)$ & $53.49(5.6)$ & $29.69(5.4)$ & $29.19(6.3)$ \\
Cubic & $\mathbf{3 . 4 1}(\mathbf{1 . 5})$ & $33.38(7.4)$ & $52.30(6.4)$ & $32.81(5.09)$ & $31.06(5.8)$ \\
$I b_{K}(\mathrm{k}=3)$ & $3.43(1.3)$ & $\mathbf{3 0 . 5 5 ( 1 1 . 3 )}$ & $53.29(9.5)$ & $29.69(12.3)$ & $\mathbf{2 7 . 6 3 ( 7 . 9 )}$ \\
\hline ID3 & $5.86(2.5)$ & $35.65(8.3)$ & $\mathbf{4 5 . 7 0 ( 6 . 7 )}$ & $\mathbf{2 9 . 0 6 ( 1 1 . 7 )}$ & $32.06(9.6)$ \\
C4.5 & $5.43(1.9)$ & $39.81(10.2)$ & $51.09(3.5)$ & $31.56(11.5)$ & $34.16(8.7)$ \\
\hline PART & $5.29(1.9)$ & $38.42(8.5)$ & $55.88(7.8)$ & $42.51(10.12)$ & $34.78(8.2)$ \\
\hline BNN & $4.01(2.4)$ & $36.11(9.8)$ & $56.48(5.2)$ & $32.81(9.3)$ & $29.51(9.7)$ \\
SMO & $\mathbf{3 . 4 3 ( 1 . 6 )}$ & $\mathbf{3 1 . 4 8 ( 1 1 . 1 )}$ & $\mathbf{4 4 . 1 1 ( 5 . 6 )}$ & $\mathbf{2 9 . 6 8 ( 1 1 . 6 )}$ & $\mathbf{2 5 . 1 5 ( 5 . 2 )}$ \\
\hline
\end{tabular}

Table 3: GE configuration.

\begin{tabular}{|l|l|}
\hline Parameter & Value \\
\hline Generation & 500 \\
\hline Population & 1000 \\
\hline Ending & $0.95 \%$ of the ideal fitness \\
\hline Operators & $\begin{array}{l}\text { Prob. Cross (0.8) } \\
\text { Prob. Repro. (0.2) } \\
\text { Prob. Mutation (0.3) } \\
\text { Max. Wrappring (2) }\end{array}$ \\
\hline Selection & Tournament (2) \\
\hline \# Codons & 200 codons \\
\hline Evaluation & $\begin{array}{l}\text { Eq. 3 with statistics from the } \\
\text { level-1 outputs }\end{array}$ \\
\hline Initialization & Ramped \\
\hline Replacement & Steady-State (SS) \\
\hline Random Seed & 10 \\
\hline
\end{tabular}

Figure 2 represents the grammar that defines the genotype to fenotype mapping process used to transform the individual into a level-1 model. At the end of the GE training process, the individual with the best fitness will be selected as the level-1 model.

The fitness of individuals is computed by equation 3 , which is based on the statistic about the accuracy rate, and unclassified rate from the level-1 outputs:

$$
\text { fitness }=0.75 \cdot \text { accuracy }-0.25 \cdot \text { unclassified }
$$

Each component has an associated weighting value that models the individual's behaviour. Finally, table 3 contains the GE configuration used in the metalearning searching process.

\subsection{Training and Testing the Models}

Given a dataset $\alpha=\left\{\left(y_{n}, x_{n}\right), n=1 . . N\right\}$, where $y_{n}$ is the class value and $x_{n}$ represents the attribute values of the $n$th instance. The samples are randomly split into $J$ equal parts $\alpha_{1}, \ldots, \alpha_{J}$. Let's define $\alpha_{j}^{\text {test }}$ and $\alpha_{j}^{\text {train }}=\alpha-\alpha_{j}^{\text {test }}$ to be the test and training sets for the $j$ th fold of a J-fold cross-validation. Also, $\alpha_{j}^{\text {train }}$ is split into $M$ equal parts $\beta_{1}, \ldots, \beta_{M}$. Let's define $\beta_{j, m}^{\text {test }}$ and $\beta_{j, m}^{\text {train }}=\alpha_{j}^{\text {train }}-\beta_{j, m}^{\text {test }}$ to be the test and training sets for the $m$ th fold of another M-fold cross-validation.

Training MGE consists of M training subcycles of the level-0 models. Each one uses $\beta_{j, m}^{\text {train }}$ as level- 0 data and $\beta_{j, m}^{\text {test }}$ to test the level-0 model. The probabilities of belonging to each class are used as level-1 data for the MGE individual that is being evaluated to obtain the statistics. The average of the M statistics allows the system to compute the fitness using equation 3. The MGE final statistics are computed in a $\mathrm{J}$ fold cross-validation, which implies training MGE with $\alpha_{j}^{\text {train }}$ and testing with $\alpha_{j}^{\text {test }} \mathrm{J}$ times.

It is obvious that this training and testing process is computationally expensive as the evaluation of one individual implies several runs of the level-0 models. Nevertheless, this penalization can be avoided if all the predictions resulting from all $\beta_{j, m}^{\text {train }}$ and $\beta_{j, m}^{\text {test }}$ $\forall j i n 1 \ldots J \forall \min 1 \ldots M$ are previously computed. Therefore, the individual's evaluation only implies the replacement of the precomputed predictions into the expression, and running time is drastically decreased.

This way of training and testing the system warrants that test folds are independent, and the individual found by MGE is tested using samples that have not been used in the training process.

\section{RESULTS AND DISCUSSION}

Tables 4, 5, 6, 7 and 8 show the error rate (percentage of missclassifications) and the standard deviation for the datasets of Table 1 using the experimental set described in subsection 4.4. Also, we have applied Bagging (Breiman, 1996a), AdaBoostM1 (Freund and Schapire, 1996) and Stacking (Wolpert, 1990) from Weka in order to compare their results.

The results can be analyzed from two points of view: the improvement of meta-classifiers in compar- 
ison with the single classifiers, and the MGE improvement with respect to the other meta-classifiers

The results of a meta-classifier are related with the results of its level-0 models. Comparing tables 5 - 8 with table 2 , we observe that the meta-classifier approaches do not improve the error rate. Wisconsin dataset is the exception because the MGE approach (table 4) improves the results of the level-0 predictions (table 2). This happens because this problem is less complex than the others. Also, we have applied a t-test student between the best single-classifiers and the MGE results, and the improvements are not statistical significant (at $95 \%$ confidence level) ${ }^{12}$.

Table 4: \% of error and dev. in Wisconsin.

\begin{tabular}{|l|l|l|}
\hline Meta-level & Level-0 models & Error \\
\hline MGE & Euc., Cub., SMO & $\mathbf{2 . 7 2}(\mathbf{1 . 7})$ \\
\hline Bagging & $I b_{3}$ & $3.43(1.6)$ \\
Bagging & BNN & $4.01(2.5)$ \\
Bagging & SMO & $3.43(1.9)$ \\
\hline AdaBoost & $I b_{3}$ & $3.14(1.8)$ \\
AdaBoost & BNN & $4.86(1.9)$ \\
AdaBoost & SMO & $3.43(1.7)$ \\
\hline Sta- $I b_{3}$ & $I b_{3}$, BNN, SMO & $3.57(1.7)$ \\
Sta-BNN & $I b_{3}$, BNN, SMO & $3.29(1.4)$ \\
Sta-SMO & $I b_{3}$, BNN, SMO & $3.57(1.5)$ \\
\hline
\end{tabular}

Table 5: \% of error and dev. in $\mu \mathrm{Ca}$.

\begin{tabular}{|l|l|l|}
\hline $\begin{array}{l}\text { Meta- } \\
\text { level }\end{array}$ & Level-0 models & Error \\
\hline MGE & Ham., IB3, SMO & $\mathbf{3 2 . 3 3 ( 9 . 7 )}$ \\
\hline Bagging & $I b_{3}$ & $33.79(10.3)$ \\
Bagging & $\mathrm{ID3}$ & $35.68(10.8)$ \\
Bagging & $\mathrm{SMO}$ & $33.33(12.1)$ \\
\hline AdaBoost & $\mathrm{I} b_{3}$ & $33.79(11.7)$ \\
AdaBoost & $\mathrm{ID3}$ & $36.11(9.9)$ \\
AdaBoost & $\mathrm{SMO}$ & $32.48(11.1)$ \\
\hline Sta- $\mathrm{I} b_{3}$ & $\mathrm{I} b_{3}, \mathrm{ID} 3, \mathrm{SMO}$ & $39.81(11.2)$ \\
Sta-SMO & $\mathrm{I} b_{3}, \mathrm{ID} 3, \mathrm{SMO}$ & $35.64(10.5)$ \\
\hline
\end{tabular}

Table 6: $\%$ of error and dev. in DDSM.

\begin{tabular}{|l|l|l|}
\hline Meta-level & Level-0 models & Error \\
\hline MGE & Clk., ID3, SMO & $47.10(5.6)$ \\
\hline Bagging & C4.5, & $51.29(4.5)$ \\
Bagging & ID3 & $49.91(6.4)$ \\
Bagging & SMO & $44.91(6.3)$ \\
\hline AdaBoost & C4.5 & $53.89(8.3)$ \\
AdaBoost & ID3 & $46.31(4.3)$ \\
AdaBoost & SMO & $\mathbf{4 2 . 7 2 ( 5 . 7 )}$ \\
\hline Sta-C4.5 & C4.5, ID3, SMO & $46.31(6.2)$ \\
Sta-SMO & C4.5, ID3, SMO & $50.49(5.9)$ \\
\hline
\end{tabular}

\footnotetext{
${ }^{1}$ Sta-XXX means that stacking is applied using XXX as meta-classifier. ID3 is not supported as meta-classifier in Weka tool because it only works with nominal values

${ }^{2}$ The CBR approach is not supported in Weka tool. We apply the next best level- 0 model
}

Table 7: \% of error and dev. in MIAS-Birads.

\begin{tabular}{|l|l|l|}
\hline Meta-level & Level-0 models & Error \\
\hline MGE & $\mathrm{Clk}, I b_{3}, \mathrm{SMO}$ & $32.50(10.6)$ \\
\hline Bagging & $\mathrm{ID} 3$ & $34.37(10.8)$ \\
Bagging & $\mathrm{I} b_{3}$ & $\mathbf{2 9 . 7 1}(\mathbf{1 1 . 9})$ \\
Bagging & $\mathrm{SMO}$ & $30.93(12.8)$ \\
\hline AdaBoost & $\mathrm{ID} 3$ & $38.43(5.9)$ \\
AdaBoost & $\mathrm{I} b_{3}$ & $32.82(11.2)$ \\
AdaBoost & $\mathrm{SMO}$ & $33.12(11.6)$ \\
\hline Sta- $I b_{3}$ & $\mathrm{ID} 3, I b_{3}, \mathrm{SMO}$ & $40.93(11.8)$ \\
Sta-SMO & $\mathrm{ID} 3, I b_{3}$, SMO & $33.43(10.4)$ \\
\hline
\end{tabular}

Table 8: $\%$ of error and std. in MIAS-3C.

\begin{tabular}{l|l|l|}
\hline Meta-level & Level-0 models & Error \\
\hline MGE & Clk., ID3, SMO & $28.57(4.6)$ \\
\hline Bagging & $I b_{3}$ & $30.12(6.8)$ \\
Bagging & ID3 & $30.74(8.8)$ \\
Bagging & SMO & $\mathbf{2 3 . 9 1 ( 5 . 1 )}$ \\
\hline AdaBoost & $I b_{3}$ & $29.81(8.8)$ \\
AdaBoost & ID3 & $38.19(6.2)$ \\
AdaBoost & SMO & $26.31(5.1)$ \\
\hline Sta- $I b_{3}$ & $I b_{3}$ ID3 SMO & $33.85(7.1)$ \\
Sta-SMO & $I b_{3}$ ID3 SMO & $31.98(6.3)$ \\
\hline
\end{tabular}

Figure 3: Meta-level discovered in Wisconsin.



Figure 4: Meta-level discovered in $\mu \mathrm{Ca}$.



Figure 5: Meta-level discovered in DDSM.

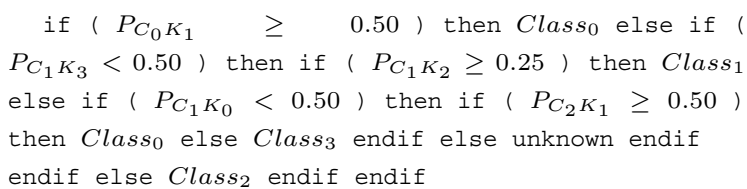

Figure 6: Meta-level discovered in MIAS-Birads.

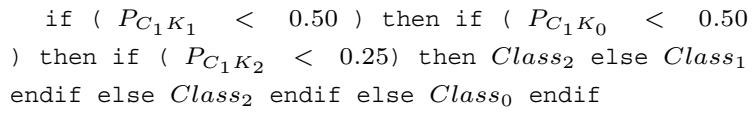

Figure 7: Meta-level discovered in MIAS-3C. 
On the other hand, the MGE results compared with the other meta-algorithm results can be considered good (although not statistically significant) as MGE almost always gets the lowest error, and the lowest standard deviation. For these reasons, the MGE results can be considered a little robuster than the others, and the MGE application provides the user more confidence on the results. The improvement of the MGE results is related to the number of attributes of the datasets. In Wisconsin and $\mu \mathrm{Ca}$ datasets, the MGE provides the best results, but in MIAS and DDSM datasets they are similar to the other methods. As a further work we could study if training GE for a higher number of generations could improve our results with MIAS and DDSM datasets.

Finally, figures 3, 4, 5, 6 and 7 show the meta-levels found by MGE. The code $P_{C_{X} K_{Y}}$ means the probability of belonging to the class $X$ of the classifier $Y$.

\section{CONCLUSIONS AND FURTHER WORK}

Meta-learning can be seen as a black-box that learns from outputs generated by other learners, in order to make an improved prediction with a higher confidence level. One of the most difficult tasks is the design of this black-box. For this reason, we propose MGE as an automatic way to define the relationships between level-0 models. MGE uses the GE approach, which is an EC approach based on a BNF grammar that leads the search process.

We have tested MGE over different breast cancer datasets and compared them with other meta-learning classifiers. Although the t-test did not find statistical differences (at 95\% confidence level), MGE almost always provided the lowest error and the lowest standard deviation. Therefore, MGE can be considered robuster than the others. Another important feature of MGE is that it can be easily tuned changing the BNF grammar without modifying the program, in order to set new ways of searching for relationships between the level-0 models. Also, MGE is adaptable to the datasets but Bagging, Adaboost and Stacking are not. Therefore, MGE can be used as a decision support system to help experts in breast cancer diagnosis to reinforce their opinion about the type of sample they are analizying.

Further work should deepen into the study of alternative ways of evaluating individuals and how they contribute to different level-0 models. Also other type of grammars could lead to different combinations of level-0 models. Adapting MGE to a distributed decision support system using a multiagent approach could benefit cost and could potentially give better results. Finally, we plan to enhance the study to other datasets.

\section{ACKNOWLEDGEMENTS}

The authors acknowledge the support provided under grant numbers TIC2002-04160-C02-02, TIC200204036-C05-03, TIN2005-08386-C05-04, 2005FIR 00237 and 2002 SGR-00/55. Also, we would like to thank Enginyeria i Arquitectura La Salle of Ramon Llull University for their support to our research group.

\section{REFERENCES}

Aamodt, A. and Plaza, E. (1994). Case-based reasoning: Foundations issues, methodological variations, and system approaches. IA Communications, 7:39-59.

Aha, D. and Kibler, D. (1991). Instance-based learning algorithms. Machine Learning, 6:37-66.

Ali, K. M. and Pazzani, M. J. (1996). Error reduction through learning multiple descriptions. Machine Learning, 24(3):173-202.

Blake, C. and Merz, C. (1998). UCI repository of machine learning databases.

Breiman, L. (1996a). Bagging predictors. Machine Learning, 24(2):123-140.

Breiman, L. (1996b). Stacked regression. Machine Learning, 24(1):49-64.

Campani, R., Bazzani, A., Bevilacqua, A., Bollini, D., and Lanconelli, N. (2000). Automatic detection of clustered microcalcifications using combined method with a support vector machine classifier. Int. Workshop on Digital Mammography.

Chan, P. K. and Stolfo, S. J. (1993). Experiments on multistrategy learning by meta-learning. In $C I K M$ ' 93 : Proceedings of the second international conference on Information and knowledge management, pages 314323, New York, NY, USA. ACM Press.

Cheng, H., Lui, Y., and Freinanis, R. (1998). A novel approach to microcalficiations detection using fuzzy logic technique. IEEE Transaction on Medical Imaging, pages 442-450.

Edwards, D., Kupinski, M., Nagel, R., Nishikawa, R., and Papaioannou, J. (2000). Using a bayesian neural network to optimally eliminate false-positive microcalcifications detection in a cad scheme. Int. Workshop on Digital Mammography.

Flann, N. S. and Dietterich, T. G. (1989). A study of explanation-based methods for inductive learning. Machine Learning, 4(2):187-226.

Fornells, A., Camps, J., Golobardes, E., and Garrell, J. (2005a). Comparison of strategies based on evolutionary computation for the design of similarity functions. 
In Artificial Intelligence Research and Development, pages 231-238. IOS Press.

Fornells, A., Camps, J., Golobardes, E., and Garrell, J. (2005b). Incorporación de conocimiento en forma de restricciones sobre algoritmos evolutivos para la búsqueda de funciones de similitud. In IV Congreso Español sobre Metaheurísticas, Algoritmos Evolutivos y Bioinspirados, MAEB'2005, pages 397-404. Thomson.

Frank, E. and Witten, I. H. (1998). Generating accurate rule sets without global optimization. In ICML '98: Proceedings of the Fifteenth Int. Conference on Machine Learning, pages 144-151. Morgan Kaufmann Publishers Inc.

Freeman, J. A. and Skapura, D. M. (1991). Neural Networks: Algorithms, Applications and Programming Techniques. Addison Wesley.

Freund, Y. and Schapire, R. E. (1996). Experiments with a new boosting algorithm. In International Conference on Machine Learning, pages 148-156.

Garrell, J., Golobardes, E., Bernadó, E., and Llorà, X. (1999). Automatic diagnosis with genetic algorithms and case-based reasoning. AI in Engineering, 13(4):362-367.

Goldberg, D. E. (1989). Genetic Algorithm in Search, Optimization, and Machine Learning. Addison-Wesley.

Golobardes, E., Llorà, X., Salamó, M., and Martí, J. (2002). Computer aided diagnosis with case-based reasoning and genetic algorithms. Journal of Knowlegde Based Systems, pages 45-52.

Golobardes, E., Nieto, M., Salamó, M., J.Camps, Calzada, G., Martí, J., and Vernet, D. (2001). Generació de funcions de similitud mitjançant la programació genètica pel raonament basat en casos. CCIA, 25:100-107.

Heath, M., Bowyer, K., Kopans, D., Moore, R., and Kegelmeyer, P. (2000). The digital database for screening mammography. Int. Workshop on Dig. Mammography.

Kauffman, G., Salfity, M., Granitto, P., and Ceccato, H. (2000). Automated detection and classification of clustered microcalcifications using morphological filtering and statistical techniques. International Workshop on Digital Mammography.

Koza, J. R. (1992). Genetic Programming. Programing of computers by means of natural selection. MIT Press.

Kwok, S. W. and Carter, C. (1990). Multiple decision trees. In UAI '88: Proceedings of the Fourth Annual Conference on Uncertainty in Artificial Intelligence, pages 327-338. North-Holland.

Martí, J., Español, J., Golobardes, E., Freixenet, J., García, R., and Salamó, M. (2000). Classification of microcalcifications in digital mammograms using case-based reasonig. Int. Workshop on Digital Mammography.

Oliver, A., Freixenet, J., Bosch, A., Raba, D., and Zwiggelaar, R. (2005a). Automatic classification of breast tissue. Iberian Conference on Pattern Recognition and Image Analysis, pages 431-438.
Oliver, A., Freixenet, J., and Zwiggelaar, R. (2005b). Automatic classification of breast density. IEEE International Conference on Image Processing. to appear.

Oliver, J. J. and Dowe, D. L. (1995). On pruning and averaging decision trees. In Proceedings 12th Int. Conf. Machine Learning, pages 430-437. Morgan Kaufmann.

Platt, J. (1998). Fast training of support vector machine using sequential minimal optimizations. In Schölkipf, B., Burges, C., and Smola, A., editors, Advances in Kernel Methods-Support Vector Learning, Cambridge, M.A. MIT PRess.

Quinlan, J. R. (1986). Induction of decision trees. Mach. Learn., 1(1):81-106.

Quinlan, R. (1993). C4.5: Programs for Machine Learning. Morgan Kaufmann Publishers.

Ryan, C., Collins, J. J., and O’Neill, M. (1998). Grammatical evolution: Evolving programs for an arbitrary language. In Proceedings of the First European Workshop on Genetic Programming, volume 1391, pages 83-95. Springer-Verlag.

Samuels, T. H. (1998). Illustrated Breast Imaging Reporting and Data System BIRADS. American College of Radiology Publications, 3rd edition.

Schapire, R. E., Freund, Y., Bartlett, P., and Lee, W. S. (1997). Boosting the margin: a new explanation for the effectiveness of voting methods. In Proc. 14th International Conference on Machine Learning, pages 322-330. Morgan Kaufmann.

Suckling, J., Parker, J., and Dance, D. (1994). The mammographic image analysis society digital mammogram database. In Gale, A., editor, Proc. 2nd Internat. Workshop on Digital Mammography, pages 211-221.

Ting, K. M. and Witten, I. H. (1997a). Stacked generalizations: When does it work? In IJCAI, pages 866-873.

Ting, K. M. and Witten, I. H. (1997b). Stacking bagged and dagged models. In Proc. 14th Int. Conference on Machine Learning, pages 367-375. Morgan Kaufmann.

Vallespí, C., Golobardes, E., and Martí, J. (2002). Improving reliability in classification of microcalcifications in digital mammograms using case-based reasoning. In Proceedings of the 5th Catalonian Conference on AI: Topics in Artificial Intelligence, Lecture Notes In Computer Science, volume 2504, pages 101112 , London, UK. Springer-Verlag.

Winfields, D., Silbiger, M., and Brown, G. (1994). Technology transfer in digital mamography. Report of the Joint National Cancer Institute, Workshop of May 1920, Invest. Radiol, pages 507-515.

Witten, I. H. and Frank., E. (2000). DataMining: Practical machine learning tools and techniques with Java implementations. Morgan Kaufmann Publishers.

Wolpert, D. H. (1990). Stacked generalization. Technical Report LA-UR-90-3460, The Santa Fe Institute, New Mexic. 